\title{
Jules Ferry et l'école rurale
}

\section{Pierre Barral}

\section{OpenEdition}

Journals

Édition électronique

URL : http://journals.openedition.org/trema/1836

DOI : 10.4000/trema.1836

ISSN : 2107-0997

\section{Éditeur}

Faculté d'Éducation de l'université de Montpellier

\section{Édition imprimée}

Date de publication : 1 décembre 1997

Pagination : 7-16

ISSN : 1167-315X

\section{Référence électronique}

Pierre Barral, « Jules Ferry et l'école rurale », Tréma [En ligne], 12-13 | 1997, mis en ligne le 01

décembre 1997, consulté le 19 avril 2019. URL : http://journals.openedition.org/trema/1836 ; DOI :

10.4000/trema.1836

Ce document a été généré automatiquement le 19 avril 2019

Trema 


\title{
Jules Ferry et l'école rurale
}

\author{
Pierre Barral
}

1 Le nom de Jules Ferry, après un siècle écoulé, reste connu de tous les Français pour son rôle dans la refonte des institutions scolaires au début de la Troisième République. Installé à Paris, bourgeois de culture citadine, il revenait volontiers dans le pays lorrain de son enfance, y contemplait la nature, y écoutait les hommes de la terre. Essayons de préciser, à partir de ses discours publics et de ses notes privées, comment il voyait la situation antérieure de l'école de campagne, quel projet propre il développa pour elle, quelles missions il lui assignait. En corrigeant les amplifications et les déformations du mythe, cet examen permet de mieux cerner la réalité des faits et des idées.

\section{L'école rurale avant Ferry}

2 L'instruction populaire est plus ancienne en France, à la campagne comme à la ville, que le montrent certains clichés simplistes. Sous l'Ancien Régime, beaucoup de villages possédaient déjà leur école, suscitée et contrôlée par l'Église catholique. La Révolution forma des projets ambitieux, qui en fait n'aboutirent guère. Ce fut la Monarchie de Juillet qui établit un réseau efficace, par l'impulsion de Guizot. A ce grand précurseur, Jules Ferry rend plusieurs fois hommage. "J'estime, quant à moi, » déclare-t-il le 27 novembre 1880, "que la loi de 1833, qui a fait de l'entretien de l'école une obligation communale, a donné à toutes les communes de France une leçon de civilisation qui a produit des fruits magnifiques : elle leur a appris le devoir social... Écrire le devoir social au budget, c'est faire l'éducation des populations rurales». Sous le Second Empire, Victor Duruy créa le certificat d'études et étendit l'obligation aux écoles de filles. Ainsi, enregistre Ferry, sur 36000 communes, " en 1837 il y avait 5667 communes dépourvues d'école; en 1877 il n'y en a plus que 312 ». Et il s'en félicite: "ce sont là, Messieurs de beaux résultats». En outre son prédécesseur Agénor Bardoux, du centre gauche, l'aïeul de Valéry Giscard d'Estaing, vient de dégager en 1878 des subventions pour constructions. L'historien Antoine Prost commente : "le législateur républicain hérite en réalité de l'effort de tout un siècle. Un vaste mouvement de scolarisation qu'aucun ministre n'aurait pu susciter a presque recouvert la France entière ${ }^{1}$ 
3 L'application de la règle pouvait certes laisser à désirer, car l'installation matérielle des classes dépendait des conseils municipaux, qu'on dit toujours soucieux au village de limiter la charge des impositions. Les inspecteurs se plaignirent souvent des bâtiments délabrés, du poêle qui fumait, des latrines sales. L'équipement pédagogique restait fort sommaire. Ainsi en Thiérache, dans la jeunesse d'Ernest Lavisse, il n'y avait «ni cartes ni tableaux noirs ni tables ni pupitres » : l'élève écrivait sur une planchette de bois posée sur ses genoux. Ces témoignages sont relevés dans l'œuvre brillante d'Eugen Weber, La fin des terroirs $^{2}$ (version française, Fayard, 1983), qui à mon avis, tend à exagérer l'archaïsme de la société paysanne au XIXe siècle. La vulgate républicaine se plait à peindre d'une palette sombre toutes les déficiences des anciennes écoles communales: les manuels primaires reproduisent, avec des remarques péjoratives, la sinistre gravure publiée par L'Illustration en 1872, (Une école dans le Jura). L'écho en émerge encore dans le téléfilm de Jean-Michel Gaillard sur Jules Ferry (1993).

4 On ne saurait cependant généraliser de telles observations, qui valent seulement pour certaines régions plus isolées du Centre et de l'Ouest. Un solide érudit lorrain, retraité de l'enseignement, Pierre Moinaux s'est attaché, avec une ténacité parfois âpre, à dénoncer " ces images pittoresquement misérabilistes, auxquelles s'est alimenté le mythe dichotomiste d'un avant et d'un après-Jules Ferry parfaitement tranché ». Dans une brochure aux photographies abondantes, L'école dans la région de Saint-Diéé, il convainc ses lecteurs que les communes rurales des Vosges ont édifié dès 1830 des bâtiments scolaires convenables et qu'elles les ont agrandis au long du siècle. Maires et conseillers n'hésitaient pas à y engager des dépenses appréciables afin d'assurer à leurs enfants une bonne éducation de base. Or il s'agit là du pays même où naquit le ministre républicain, de la circonscription qui l'élut à la Chambre pendant dix-huit ans. Il loue lui-même cette situation favorable le 20 décembre 1880 : «Et les Vosges? Chacun sait qu'elles occupent un rang très honorable dans la statistique de l'enseignement primaire».

On connaît d'autre part l'enquête d'ensemble lancée vers 1875 par un chercheur original, Louis Maggiolo. Cet autre Lorrain, né à Nancy d'un père génois, recteur en retraite, a su mobiliser des centaines d'instituteurs, essentiellement ruraux, pour compter la proportion des conjoints ayant signé leur acte de mariage dans toutes les régions de France et en quatre tranches quinquennales échelonnées sur deux siècles. Le critère, aisé à mettre en œuvre, peut sembler trop rudimentaire: sans maitriser l'écriture, certains sont capables de dessiner leur signature, comme le montre une jolie scène du film de Noël Vigne, Le retour de Martin Guerre. La méthode néanmoins fait ressortir des contrastes significatifs entre les époques, entre les lieux et entre les sexes. Cette précieuse documentation, qui avait été oubliée, a été signalée en 1957 par la revue Population,puis cartographiée et analysée avec bonheur par François Furet et Jacques Ozouf ${ }^{4}$. Pour Maggiolo, notent-ils justement, "l'alphabétisation n'était qu'un signe de l'école, un indicateur de l'activité scolaire et pédagogique ». A la fin du XVII siècle un homme sur trois et une femme sur huit savaient signer leur nom. Les proportions s'accrurent au cours du XVIII ${ }^{\mathrm{e}}$ et le mouvement s'accéléra au XIX ${ }^{\mathrm{e}}$ : «le progrès », se réjouissait l'initiateur de l'enquête, " de 1790 à 1875-77 a été de 34 sur 100 pour les hommes et de 44 sur 100 pour les femmes. Il n'est pas étonnant qu'un siècle qui a fait plus d'efforts pour répandre l'instruction primaire ait obtenu plus de résultats ». D'autre part, les différences régionales sont fortement marquées et la ligne Saint Malo-Genève, avec un appendice en Dauphiné, constitue une frontière culturelle devenue aujourd'hui classique. "Grossièrement », concluent Furet et Ozouf, «la France du Nord et du Nord-Est sait lire et écrire à la fin du XVIII siècle, alors que l'autre France entreprend 
seulement de rattraper son retard". Ces observations sont d'ailleurs confirmées par le classement des conscrits lors de leur incorporation dans l'armée comme par le tableau de certaines réponses au recensement général de 1872.

6 Au début de la Troisième République, il ne s'agit donc pas de créer l'école rurale, car elle existe partout et son équipement est en cours d'amélioration. Mais son fonctionnement est miné de l'intérieur par l'ampleur de l'absentéisme. C'est là l'axe critique du bilan dressé par le ministre le 20 novembre 1880. Tout d'abord, «le semestre d'été est le moment de la désertion ». Avant et après le temps des vacances réglementaires, les familles font travailler les enfants selon les urgences, à la fenaison, aux vendanges, à la récolte des pommes de terre, à la garde du troupeau. Les efforts de l'administration scolaire n'ont pu qu'élever peu à peu le "coefficient d'assiduité", c'est-à-dire "le rapport entre le nombre d'élèves présent pendant le semestre d'été et le nombre d'élèves présent pendant le semestre d'hiver », de $48 \%$ en 1834, à $62 \%$ en 1843, à $79 \%$ en 1877. Ensuite «l'enfant quitte l'école trop tôt », car "l'immense majorité des populations de nos écoles ne suit l'école que de l'âge de six à onze ans"; dès que les enfants deviennent plus vigoureux et plus utilisables, ils doivent aider leurs parents au travail sans interruption et ils deviennent à titre définitif des "déserteurs de l'école ».

7 Jules Ferry a la ferme volonté de remédier à ce fléau en transférant sur les pères de famille la règle légale qui concernait jusqu'alors la commune. Il fait voter en ce sens la loi fondamentale du 28 mars 1882 sur l'obligation scolaire jusqu'à 13 ans. Antoine Prost peut qualifier cette mesure comme signifiant "essentiellement une prolongation de la scolarité effective " ${ }^{5}$ 'L'article 12 prévoit des sanctions, si les enfants manquent la classe sans raison valable plus de quatre demi-journées en un mois.

8 En fait, on ne parvient pas à les mettre en œuvre devant la résistance sourde des familles rurales, et si l'assiduité s'améliore, les variations saisonnières s'observeront encore longtemps. La gratuité, instaurée dans le même élan, doit contribuer à "rendre la fréquentation des écoles plus facile», à "peupler les écoles dans les villages». Beaucoup de villes la pratiquaient déjà et le 13 juillet 1880 le ministre plaçait cette disposition « au premier rang ", "parmi les inégalités d'avantages et de conditions qui mettent le séjour des villes tellement au dessus du séjour des campagnes, et qui sont certainement des causes de la dépopulation des campagnes, de leur abandon pour les villes». Sa généralisation par la loi doit retenir au village les parents de condition modeste les plus légitimement soucieux de l'instruction de leurs enfants.

\section{Le projet universaliste}

9 A plusieurs reprises Jules Ferry a exposé avec solennité une conception forte de l'école primaire publique. On cite souvent ces grands textes, qui sont devenus des références classiques. A l'origine, le programme du jeune député parisien, salle Molière, le 10 avril 1870, contenant le fameux serment, consacrer à l'éducation du peuple «tout ce que j'ai d'âme, de coeur, de puissance physique et morale ». Au pouvoir, les discours parlementaires de 1879-1881 et la célèbre circulaire aux instituteurs, du 17 novembre 1883. Enfin, au terme de sa carrière politique, le bilan rétrospectif de l'œuvre accomplie, le 6 Juin 1889. J'ai repris ce corpus, pour y scruter l'attention portée à l'école rurale.

10 A cette relecture, j'ai d'abord été déconcerté de constater que le projet du ministre de l'Instruction Publique comporte un caractère général et ne s'articule pas selon les 
situations particulières. Il considère l'élève dans une optique globale, sans faire de différence entre les garçons et les filles, entre les petits ruraux et les petits citadins, entre les enfants des familles croyantes et les enfants des familles anticléricales. Dans cet esprit, le discours de la salle Molière, proposant "l'égalité d'éducation ", appelle même à effacer la séparation "entre ceux qui ont reçu l'éducation et ceux qui ne l'ont pas reçue», comme constituant entre les classes sociales la distinction "fondamentale, et d'autant plus difficile à déraciner ». Il se masque le cloisonnement des lycées et collèges qui, à cette époque, éloigne en fait les fils de la bourgeoisie du réseau de l'instruction populaire. Et il présente comme l'idéal à atteindre « le premier rapprochement, la première fusion qui résulte du mélange des riches et des pauvres sur les bancs de quelque école».

11 Les vues du grand Vosgien s'insèrent dans la conception universaliste de l'égalité, définie par la Révolution française, qui contraste avec la valeur prépondérante de la liberté dans la tradition britannique et la solidarité communautaire de la tradition allemande. Sa veuve a justement saisi le sens de son action quand elle a inspiré le sujet central du monument érigé a Saint-Dié, peu après sa mort. La République, levant les yeux vers l'homme d'État, présente l'un à l'autre deux enfants : un écolier français, en blouse et sabots, et un écolier " annamite " (comme on disait alors), en costume national et pieds nus. Scène symbolique de la priorité de l'instruction pour tous. Si nos contemporains, exaltant l'instruction populaire et condamnant l'expansion coloniale, opposent volontiers aujourd'hui les deux thèmes de la politique ferryste, pour Jules Ferry, le second semble de même nature que le premier. Ces slogans, gravés de part et d'autre du socle du monument, sont les faces complémentaires d'une même entreprise d'éducation qui veut mener à la conscience adulte des êtres encore dans l'enfance.

Pour l'école primaire, les dispositions de la loi et des règlements sont donc identiques sur tout le territoire national, de même que la Troisième République applique un statut uniforme des communes à la ville et à la campagne (sauf l'exception de la capitale). Divers courants agrariens et régionalistes dénonceront une formule trop abstraite, qui ne prendrait pas en compte les données réelles de la vie collective. Cependant, quand on regarde les textes de près, on ne peut valider tout à fait ce reproche. Si le discours du ministre s'abstient de proclamer des principes ruralistes, il contient bien des précisions concrètes qui font surgir le climat de la campagne. S'il évite presque constamment le terme "paysan", jugé dépréciatif, il évoque clairement le monde de la terre et sa description vaut en général davantage pour l'école du village que pour celle de la ville.

13 C'est ainsi le cas quand il salue l'effort financier des conseils municipaux pour étendre les écoles de filles au dessous du seuil des 500 habitants par commune, prévu en 1867, et plus généralement pour améliorer partout les bâtiments. Devant le Sénat il se félicite le 9 décembre $1879 \mathrm{du}$ «lien interne qui associe la vie communale aux destinées de l'enseignement primaire»:

"c'est à cette alliance étroite que sont dues, depuis sept ans, tant de choses vraiment grandes, dont les petites communes sont le théâtre [...] Dans cette accumulation de dossiers qui constituent le travail quotidien pour un ministre de l'Instruction publique, il est une case profondément attrayante... la case bienheureuse et bénie qui renferme les demandes de subvention et d'emprunt des petites communes pour l'agrandissement des écoles ".

14 Admirables apparaissent « ces belles choses, ces sacrifices volontaires, ces contributions des pauvres centimes des petits propriétaires et des petits travailleurs recueillies pour porter la lumière dans le fond de nos campagnes ». Image certes idyllique, que nuance Ferry, un peu amer, dix ans après : «l'entreprise a été coûteuse », reconnaît-il le 6 juin 
1889 et «c'est par les finances qu'on lui fait son procès devant ces masses du suffrage universel rural, tout à la fois éprises des idées d'économie et très avides de subventions ».

A l'occasion, le ministre reproche aux congréganistes de s'établir de préférence dans les grosses agglomérations (à l'exception des «Béates» de Haute-Loire), de se réserver «les écoles avantageuses, les bonnes écoles à plusieurs classes", "tandis que les mauvaises écoles, les postes pénibles, isolés et peu fructueux sont, en immense majorité, occupés par les laïques " (2août 1879). Car il perçoit bien les difficultés de fonctionnement qu'engendre la dispersion de l'habitat. "Vous savez », dit-il aux sénateurs le 2 juillet 1881, " combien sont nombreuses ces petites écoles où l'insuffisance des locaux ne permet d'établir qu'une seule classe». Il fait étudier "le meilleur moyen d'organiser dans ces localités qui, en général, n'ont pas de salles d'asile, renseignement des tout petits enfants", de "faire une petite classe dans ces écoles de village ». A celles-ci il veut étendre le programme conçu dans toute son ampleur: "nous groupons autour de renseignement fondamental $d u$ 〈lire, écrire et compter» », annonce-t-il au Congrès pédagogique de 1881, "les leçons de choses, l'enseignement du dessin, les notions d'histoire naturelle, les musées scolaires, la gymnastique, les promenades scolaires, le travail manuel de l'atelier placé à côté de l'école, le chant, la musique chorale qui y pénétreront à leur tour ". Objectifs ambitieux assurément, mais «ces accessoires feront de l'école primaire, de l'école du moindre hameau, du plus humble village, une école d'éducation libérale ».

L'effort demandé aux élèves est décrit avec une pointe d'émotion. "Il n'est pas admissible ", dit Ferry le 23 décembre 1880, "que des enfants qui ont fait trois ou quatre kilomètres par la boue, par des temps humides, pour venir à l'école n aient pas un lieu pour déposer leurs manteaux, leurs petits sabots, le petit panier dans lequel ils apportent leurs provisions». Aussi, sous les applaudissements, prescrit-il qu'on veille à installer des vestiaires et à équiper des casiers dans les écoles de village. Et quand on parle devant lui des distances à parcourir, il apprécie exactement la différence des situations : "est-ce que tous les kilomètres se valent ? quels kilomètres? sont-ce des kilomètres des Pyrénées ou de la plaine de Provence?» (23 décembre 1880).

Quand il se représente le maître, il pense essentiellement à l'instituteur rural. «Celui-ci était autrefois", dit-il sur un ton polémique, "le serviteur, on pourrait presque dire le serf de tout le monde, le serviteur de M. le maire, de M. le curé, le serviteur du château " (19 avril 1881). Il n'en est plus ainsi sous la République, mais " quelle est son épreuve ? [...] c'est l'isolement » :

" tombé dans un petit village très jeune, à l'âge où l'on n'est pas encore chef de famille, il est bien seul [...] et la solitude, c'est bien vite la lassitude de l'esprit, c'est l'ennui profond, couvrant de je ne sais quelle teinte grise et sombre le métier qu'on vient d'adopter et qui est pourtant un si noble et si grand métier. Quoi! pas d'échange d'idées, pas de livres, pas de lien. Bien vite on se lasse du travail solitaire; bien vite la pensée se fatigue, s'use, s'atrophie, quand elle ne peut se communiquer et se renouveler $"$.

18 Aussi le ministre invite-t-il les inspecteurs, au Congrès pédagogique de 1880, à «être la lumière qui périodiquement leur arrive du côté de l'orient ", à " rappeler à la vie intellectuelle ceux qui commencent à s'engourdir». Et il conclut, dans un éloge teinté de quelque flatterie paternaliste: "En insistant sur les côtés difficiles de la profession, je marque en même temps, messieurs, ce qui en fait la noblesse, car s'il n'était pas un peu méritoire d'être instituteur de campagne, ce ne serait ni si honorable ni si honoré». 


\section{Les missions de l'école laïque}

19 Pour Ferry, comme pour Gambetta et pour tous leurs compagnons de lutte, l'école apparaît comme la base de la République à fonder : il lui revient d'orienter la vie civique, et d'abord au village. Elle doit redresser les principes de la culture, les axes de la société, les règles du pouvoir. La clef en est bien sûr "la laïcité ", proclamée comme l'étoile tutélaire d'un monde nouveau. Encore faut-il cerner la signification du terme et en dégager les axes complémentaires.

L'innovation spectaculaire est la séparation de l'école et des Églises (en fait avant tout de l'Église catholique). Ceci représente une rupture radicale avec la pratique antérieure des régimes français successifs; ceci représente aussi une différenciation explicite avec la pratique des autres nations européennes qui laissent toutes alors aux courants religieux quelque influence sur la formation spirituelle des élèves. Ferry, lui, déclare le 10 juin 1881: "L'Instruction publique, qui est le premier des services publics, doit tôt ou tard être sécularisée comme l'ont été depuis 1789 et le gouvernement, et les institutions, et les lois». Sécularisation effectuée par la loi du 28 mars 1882, complétée le 30 octobre 1886. La laïcisation des programmes supprime les leçons de catéchisme, que donnait l'instituteur sous le contrôle du curé ou du pasteur. Les leçons de morale qui y sont substituées doivent ignorer toute motivation métaphysique : "la vraie morale, la grande morale, la morale éternelle c'est la morale sans épithète» (2 juillet 1881). La laïcisation du personnel implique l'exclusion des membres des congrégations, qui tenaient la majorité des écoles publiques de filles; en pratique, le recrutement de laïques qualifiées nécessite des délais et la transition se prolonge sur une génération.

21 Sur le sens profond de cette sécularisation, un débat est ouvert. Jules Ferry répète avec insistance que l'émancipation de l'Église ne comporte pas de persécution des croyances : «oui, nous avons voulu la lutte anticléricale, mais la lutte antireligieuse, jamais » (10 juin 1881) ; "l'irréligion d'État, le fanatisme à rebours, nous le réprouvons autant que vous» (11 mars 1882). De nombreux auteurs, dont Fresnette Pisani-Ferry, petite nièce de notre héros, mettent l'accent sur ces affirmations apaisantes. 


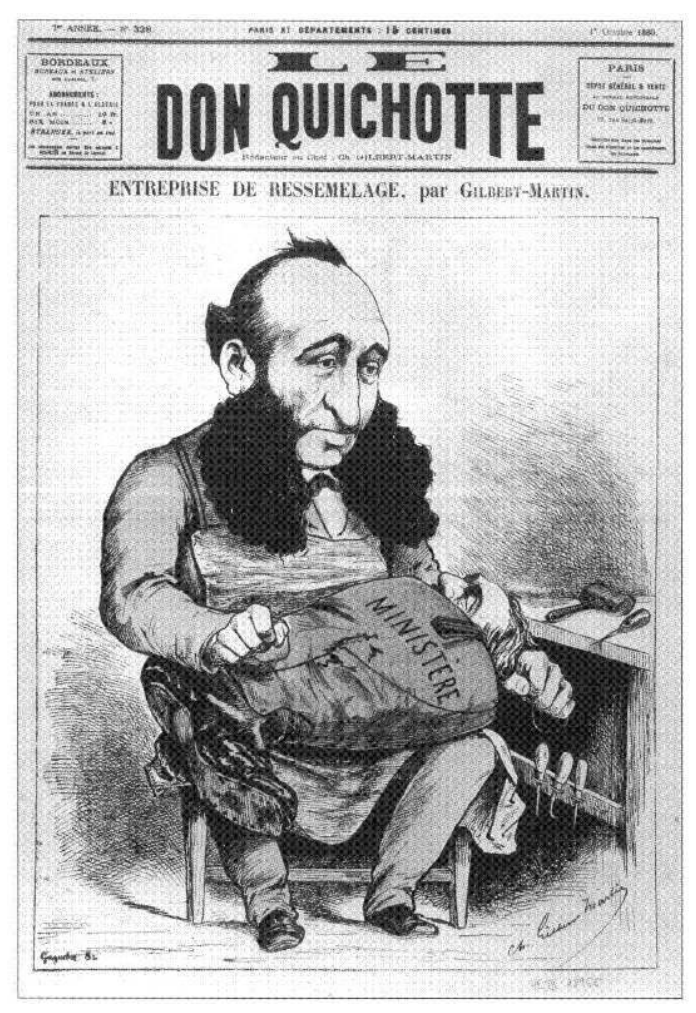

Il me semble toutefois que ce niveau du discours n'épuise pas le problème. En privé, quand il parle à son frère ou en loge maçonnique, le Vosgien considère la religion comme un aveuglement funeste de la raison. Jaurès rapportera lui avoir arraché l'aveu : « Mon but est d'organiser l'humanité sans Dieu et sans roi ", formule empruntée à Auguste Comte, qui a beaucoup marqué sa pensée. Souhaitant par conviction la disparition de la foi, il l'attend du progrès de l'instruction dans la longue durée. Non certes d'une contrainte sur les esprits, qu'il écarte par libéralisme viscéral comme par réalisme politique.

Car il perçoit bien que la France rurale reste largement attachée aux traditions religieuses. En juin 1877, il s'amuse de l'affluence aux cérémonies de la Fête-Dieu : «moi, damné authentique... je suis l'élu d'un peuple qui fait des reposoirs, qui tient à la République, mais qui ne tient pas moins à ses processions ». Le 6 juillet 1879, il combat un amendement du radical Madier de Montjau, qui voulait interdire aux congrégationistes tout enseignement, même privé : "Il y a un parti pris bien arrêté parmi les paysans de nos campagnes et dans les villages de France, de ne point souffrir... que l'Église mette le pied dans la politique... mais il y a aussi un parti non moins arrêté de laisser l'Église maitresse chez elle! » Aussi ferme-t-il les yeux quand certaines municipalités conservent le crucifix dans la salle de classe; si ce problème suscite un grave incident en 1880 , c'est à Paris, par une initiative intempestive du préfet de la Seine. Le ministre accepte même en décembre 1880 que dans des cas limites le catéchisme puisse être enseigné dans l'école, hors des heures de classe : compromis aussitôt balayé par la conjonction des extrêmes.

Comme Gambetta, Ferry mesure en effet l'enjeu électoral : la population rurale constitue encore les deux tiers de la population totale. Après la parenthèse bonapartiste, elle s'est majoritairement ralliée à la République. Pendant la crise du 16 mai, le député de Saint-Dié note: "C'est Jacques Bonhomme faisant le gros dos et serrant le poing dans ses poches, résolu à 
regarder faire, à ne rien dire, mais à porter dans l'urne le petit bulletin dont lui seul sait le secret et dont il connaît la toute puissance ». Aussi, face aux intransigeants, déclare-t-il à Périgueux en 1884 : «la République sera la République des paysans ou elle ne sera pas». Et un an après à Bordeaux, il salue cette "force immense ", " avec ses facultés propres ": " nous avons conquis le suffrage universel des campagnes: gardons-le bien, ne l'inquiétons pas, ne le lassons pas!». Il appartient à l'école de consolider cet acquis dans les générations à venir par l'instruction civique.

Selon ce discours de Bordeaux, c'est aussi sur la paysannerie que "repose la sécurité de notre société $»$. Dès novembre 1871, Ferry a proposé d'introduire à l'école normale des Vosges un cours d'économie libérale, car les élèves-maîtres

"plus tard auront la mission sacrée de montrer à ceux que les privations, la pauvreté exposent à toutes les tentations, que la constitution de la société n'est pas arbitraire... que le capital et la propriété sont légitimes et nécessaires... qu'il n'est pas plus possible de changer la propriété comme pivot du monde social qu'il n'est possible de changer le soleil comme pivot du monde cosmique ».

Le 17 juin 1874, à l'Assemblée nationale, il interpelle la droite: "Ah! Messieurs les défenseurs attitrés de la propriété, qui m'interrompez en ce moment, j'ai plus de foi que vous en sa solidité, car je sais qu'elle repose sur le couur et les bras de plus de dix millions de propriétaires »: vision schématique assurément, qui occulte l'ampleur en superficie du faire-valoir indirect. Idéalisant cette "démocratie rurale ", le ministre invite les instituteurs à fortifier au village l'entente sociale.

La promotion individuelle des meilleurs doit affermir l'harmonie. Dans chaque région, la mythologie républicaine rapporte l'anecdote édifiante du fils de famille modeste, qui est remarqué pour son intelligence par un élu républicain de passage et qui, avec l'appui de ce "délégué cantonal », parvient à mener de hautes études : cette scène symbolique ne manque pas dans le téléfilm sur Jules Ferry. Le plus souvent cette ascension suit la voie moyenne de l'école normale d'instituteurs ou de l'enseignement primaire supérieur. Pour celui-ci Ferry recommande d'éviter qu'il ne "s'isole et vise à une sorte d'existence à part ", qu'il « fût un collège dégénéré au lieu d'être une école perfectionnée " (rapport d'octobre 1881). Toutefois, des bourses sont créées pour permettre à certains, plus brillants, de devenir professeurs, avocats ou médecins, et la République fera quelque place à ces "boursiers " dans la troupe plus abondante des héritiers.

Enfin, l'école primaire est chargée d'éveiller et de nourrir le patriotisme des futurs citoyens. Le manuel d'instruction civique écrit par Paul Bert consacre son premier chapitre à «La Patrie », et particulièrement au service militaire. Dans l'Eure, on donne comme sujets du certificat d'études : aux garçons, la lettre d'un conscrit à sa mère, «pour la consoler de son départ "; aux filles, la réponse approbative de la mère. Mis en œuvre par les maitres de village, ce programme extirpe avec succès la résistance séculaire de la paysannerie au recrutement sous les armes. En une génération, les ruraux intériorisent comme un devoir inéluctable le passage au régiment, puis en 14-18 le sacrifice sanglant que commémorent les longues listes des monuments aux morts : non plus seulement dans la Lorraine de la frontière, mais tout autant en Bretagne, en Auvergne ou en Languedoc. Bien plus tard, le syndicaliste agricole Eugène Forget se rappellera son cri à la mobilisation : «Gamin que j'étais, j'ai sorti une ânerie : « Eh bien tant mieux, on va retrouver l'Alsace et la Lorraine! >... alors mon père m'a sérieusement gourmandé »". Les "hussards noirs de la République » ont bien travaillé pour la Revanche. 


\section{NOTES}

1. PROST A., L'enseignement en France, Paris, Armand Colin, 1967, p. 105.

2. WEBER E., La fin des terroirs, version française, Fayard, 1983.

3. MOINAUX P., L'école dans la région de Saint-Dié, Gérard Louis, Éd. Vagney, 1993.

4. FURET F. \& OZOUF J., Lire et écrire, Paris, Éditions de Minuit, 1977.

5. PROST A., op. cit., p. 101.

6. FORGET E., Le serment de l'unité paysanne, 1982, p. 25.

\section{RÉSUMÉS}

\section{Non disponible}

Not available

INDEX

Mots-clés : école primaire, France, IIIe république, institutions, Jules Ferry, ruralité Keywords : branch of power, primary school, rural context, rural life, third republic

\section{AUTEUR}

\section{PIERRE BARRAL}

Université Paul Valéry de Montpellier 